\title{
Multistate outbreak of Listeria monocytogenes infections linked to whole apples used in commercially produced, prepackaged caramel apples: United States, 2014-2015
}

\author{
K. M. ANGELO ${ }^{1 * \dagger} \uparrow$, A. R. CONRAD ${ }^{1} \dagger, A \cdot \mathrm{SAUPE}^{2}, \mathrm{H} \cdot \mathrm{DRAGOO}^{3}, \mathrm{~N} \cdot \mathrm{WEST}^{4}$,

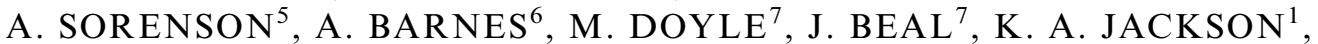 \\ S. STROIKA ${ }^{1}$, C. TARR ${ }^{1}$, Z. KUCEROVA ${ }^{1}$, S. LANCE $^{1,7}$, L. H. GOULD ${ }^{1}$, \\ M. WISE ${ }^{1}$ AND B. R. JACKSON ${ }^{1}$ \\ ${ }^{1}$ Centers for Disease Control and Prevention, Atlanta, GA, USA \\ ${ }^{2}$ Minnesota Department of Health, St. Paul, MN, USA \\ ${ }^{3}$ Arizona Department of Health Services, Phoenix, AZ, USA \\ ${ }^{4}$ New Mexico Department of Health, Santa Fe, NM, USA \\ ${ }^{5}$ Minnesota Department of Agriculture, St. Paul, MN, USA \\ ${ }^{6}$ California Department of Public Health Food and Drug Branch, Sacramento, CA, USA \\ ${ }^{7}$ Food and Drug Administration, College Park, MD, USA
}

Received 8 August 2016; Final revision 26 October 2016; Accepted 24 November 2016; first published online 9 January 2017

\section{SUMMARY}

Whole apples have not been previously implicated in outbreaks of foodborne bacterial illness. We investigated a nationwide listeriosis outbreak associated with caramel apples. We defined an outbreak-associated case as an infection with one or both of two outbreak strains of Listeria monocytogenes highly related by whole-genome multilocus sequence typing (wgMLST) from 1 October 2014 to 1 February 2015. Single-interviewer open-ended interviews identified the source. Outbreak-associated cases were compared with non-outbreak-associated cases and traceback and environmental investigations were performed. We identified 35 outbreak-associated cases in 12 states; $34(97 \%)$ were hospitalized and seven $(20 \%)$ died. Outbreak-associated ill persons were more likely to have eaten commercially produced, prepackaged caramel apples (odds ratio $326 \cdot 7$, 95\% confidence interval 32-2-3314). Environmental samples from the grower's packing facility and distribution-chain whole apples yielded isolates highly related to outbreak isolates by wgMLST. This outbreak highlights the importance of minimizing produce contamination with L. monocytogenes. Investigators should perform single-interviewer open-ended interviews when a food is not readily identified.

Key words: Foodborne infections, food safety, infectious disease epidemiology, Listeria, outbreaks.

\section{INTRODUCTION}

Listeria monocytogenes is estimated to cause over 1600 illnesses and 250 deaths annually in the United States,

\footnotetext{
* Author for correspondence: K. M. Angelo, DO, MPH-TM, 1600 Clifton Road NE, Mailstop A-38, Atlanta, GA, USA 30329-4027. (Email: kangelo@cdc.gov)

$\dagger$ These authors contributed equally to this work.
}

making it a leading cause of foodborne illness death $[1,2]$. Older adults, persons with immunocompromising conditions, and pregnant women and their neonates are at higher listeriosis risk than the general population [3, 4]. Rarely, otherwise healthy persons develop invasive listeriosis [5]. Most listeriosis is foodborne and sporadic; outbreaks have historically been linked to ready-to-eat meats and dairy products [5-8]. However, produce-associated listeriosis outbreaks are 
increasingly recognized [9], including those involving sprouts [10], stone fruit [11], celery [12], and cantaloupe [13]. Whole apples have not been implicated in listeriosis outbreaks, although apples have been recalled for possible L. monocytogenes contamination [14] and unpasteurized apple cider has been implicated as the cause of Shiga toxin-producing Escherichia coli and Cryptosporidium outbreaks [15].

On 14 November 2014, epidemiologists at the Arizona Department of Health Services and the New Mexico Department of Health notified the U.S. Centers for Disease Control and Prevention (CDC) of four listeriosis cases that occurred within the previous month, including two in otherwise-healthy children. Pulsed-field gel electrophoresis (PFGE) results were not yet available. The same day, PulseNet, the national molecular subtyping network for enteric disease surveillance, identified a listeriosis cluster with a rare PFGE pattern with cases in older adults in Minnesota (three cases) and Missouri (one case) [16, 17]. The following week, PulseNet identified a second unrelated PFGE cluster of five L. monocytogenes isolates, including the four listeriosis cases reported by Arizona and New Mexico. By 1 December, the patient count in the two clusters had risen to 22 , including a child with an L. monocytogenes cerebrospinal fluid (CSF) isolate matching one cluster and a blood isolate matching the other. Given this finding and the overlap in the temporal and geographic case distribution, investigators suspected a common source could account for cases in both clusters, and the clusters were combined into a single investigation. We describe the nationwide investigation into the infection source, including the use of real-time whole genome sequencing.

\section{METHODS}

\section{Case definition and laboratory methods}

Clinical L. monocytogenes isolates are sent to state public health laboratories for two-enzyme PFGE subtyping [18]. Since September 2013, whole genome sequencing is performed on clinical, environmental, and food L. monocytogenes isolates at state laboratories, CDC, Food and Drug Administration (FDA), and the US Department of Agriculture's Food Safety and Inspection Service. CDC analyses these sequences using whole-genome multilocus sequence typing (wgMLST), which provides superior genetic resolution compared with PFGE [11, 19]. The data have been deposited with links to BioProject accession number PRJNA212117 in the National Center for Biotechnology Information BioProject database (https://www.ncbi.nlm.nih.gov/bioproject/).

We defined an outbreak-associated case as a laboratory-confirmed L. monocytogenes infection in a US resident with an isolate cultured from a normally sterile site (e.g. blood or CSF) or from products of conception (e.g. placenta) that was highly related to other outbreak isolates by PFGE and wgMLST and collected during 1 October 2014 to 1 February 2015. Pregnancy-associated cases were defined as infection in a pregnant woman or infant (aged $\leqslant 28$ days). Medical records and death certificates were reviewed to assess whether listeriosis contributed to patients' deaths. PFGE data were shared with other countries via PulseNet International [20] for additional case finding.

\section{Epidemiological investigation}

We used several epidemiological techniques in the investigation. State and local health departments interviewed patients or surrogates using the Listeria Initiative (LI) form, which collects clinical, laboratory, demographic, and epidemiological information [21], including questions on 44 possible food exposures during the 28 days before illness onset; five questions involve produce (cantaloupe, watermelon, honeydew melon, sprouts, fruit salad). Patients were also interviewed using CDC's National Hypothesis Generating Questionnaire (NHGQ) that includes $>300$ food and restaurant exposures, and, when possible, additional open-ended interviews were performed [22]. Open-ended interviews involve lengthy, semi-structured discussions with the patient or surrogate about foods consumed during the 28-day exposure window. These interviews include assessment of the patient's typical diet and foods eaten at special events or holidays. After open-ended interviewing revealed that eight patients ate commercially produced, prepackaged caramel apples, an item not listed on the LI form or NHGQ, during their incubation periods, CDC requested that states administer a supplemental questionnaire to all recent listeriosis cases, including outbreak-associated and non-outbreak-associated cases. This supplemental questionnaire included questions about caramel apples, caramel, whole apples, and pre-cut apple slices. We compared outbreak-associated case exposures with exposures reported in the 2006-2007 FoodNet Population Survey [23] and with exposures for non-outbreak-associated listeriosis cases that 
occurred during the same time period. We calculated odds ratios (OR) and 95\% confidence intervals (CI) for exposures of interest.

\section{Traceback and environmental investigation}

FDA and state and local health and regulatory agencies performed a traceback investigation using information reported from patients about caramel apple brands and purchase locations to identify caramel apple manufacturers. Manufacturer invoices were used to determine ingredient sources. FDA and state public health and agricultural officials sampled and cultured whole apples from the distribution chain in Minnesota, Missouri, and Nebraska and sampled caramel apple production facility environments in California and Missouri to identify L. monocytogenes. After traceback investigation identified an apple grower (Grower A) that supplied the involved manufacturers, California and FDA officials inspected and sampled Grower A's apple packing facility.

\section{RESULTS}

\section{Outbreak description}

We identified 35 outbreak-associated cases in 12 US states. Most infections occurred in the southwestern United States, particularly Arizona and New Mexico, and the Midwest (Fig. 1). Eleven illnesses $(31 \%)$ were pregnancy-associated, including one fetal loss. Of non-pregnancy-associated cases, the median age was 62 years (range 7-90 years) and 33\% were female. Three children, all otherwise healthy and aged 5-15 years, developed meningitis. Thirty-four patients $(97 \%)$ were hospitalized. Listeriosis contributed to at least three of seven reported deaths (Table 1). Illness onset dates ranged from 17 October 2014 to 7 January 2015 (Fig. 2). The Public Health Agency of Canada (PHAC) identified an additional case with a patient isolate that was indistinguishable from outbreak-associated isolates by PFGE and highly related by wgMLST.

\section{Epidemiological case investigation}

No food emerged as a likely source following initial interviews with the LI form. By 10 December, ten NHGQ interviews were completed and no exposures of interest were identified; five $(63 \%)$ of eight patients reported eating apples compared with $62 \%$ of population survey respondents. Therefore, more open-ended interviews were begun. On 15 December, CDC and Texas state and local public health officials interviewed a patient's spouse; a Texas epidemiologist inquired about caramel apple consumption after noting such consumption in a previous open-ended interview. The spouse reported that the patient had eaten a caramel apples three-pack from a large retail chain. Based on this information, eight additional patients were re-contacted and all eight reported eating caramel apples. CDC deployed a supplemental questionnaire the following day.

Ultimately, 34 ill persons or surrogates were interviewed with the LI form, 12 with the NHGQ, eight with open-ended interviews, and 15 with the supplemental questionnaire; 16 patients were asked about caramel apple consumption but were not administered the supplemental questionnaire. Twenty-eight $(90 \%)$ of 31 patients with available information consumed commercially produced, prepackaged caramel apples in the 28 days before illness onset compared with one $(3 \%)$ of 36 non-outbreak-associated cases interviewed with the supplemental questionnaire during the same time period (OR 326.7, 95\% CI 32-3314). The patient from Canada also reported eating caramel apples. Of three ill persons who did not report eating caramel apples, one ate apples with caramel dipping sauce, one ate a Granny Smith apple, and one ate precut apple slices, the sources of which were unknown. Nine $(56 \%)$ of 16 people with outbreak-associated illnesses ate whole apples not part of caramel apples compared to $20(67 \%)$ of 30 non-outbreak-associated cases. Twenty-four outbreak-associated patients reported eating caramel apples purchased from a retail establishment, two purchased caramel apples at a fair, one received caramel apples from a food bank, and the source for one was unknown. Three patients named the caramel apple brand; various purchase locations were reported.

\section{Traceback and environmental investigation}

FDA and state regulatory agencies conducted a traceback investigation using caramel apple purchase locations for 11 outbreak-associated cases. These 11 locations received caramel apples from four manufacturers through multiple distributors. The four manufacturers did not share a common source of caramel, sticks, toppings (e.g. nuts, sprinkles), or packaging. However, all made caramel apples using Grower A's apples from California. FDA and California state officials inspected Grower A's apple packing facility 


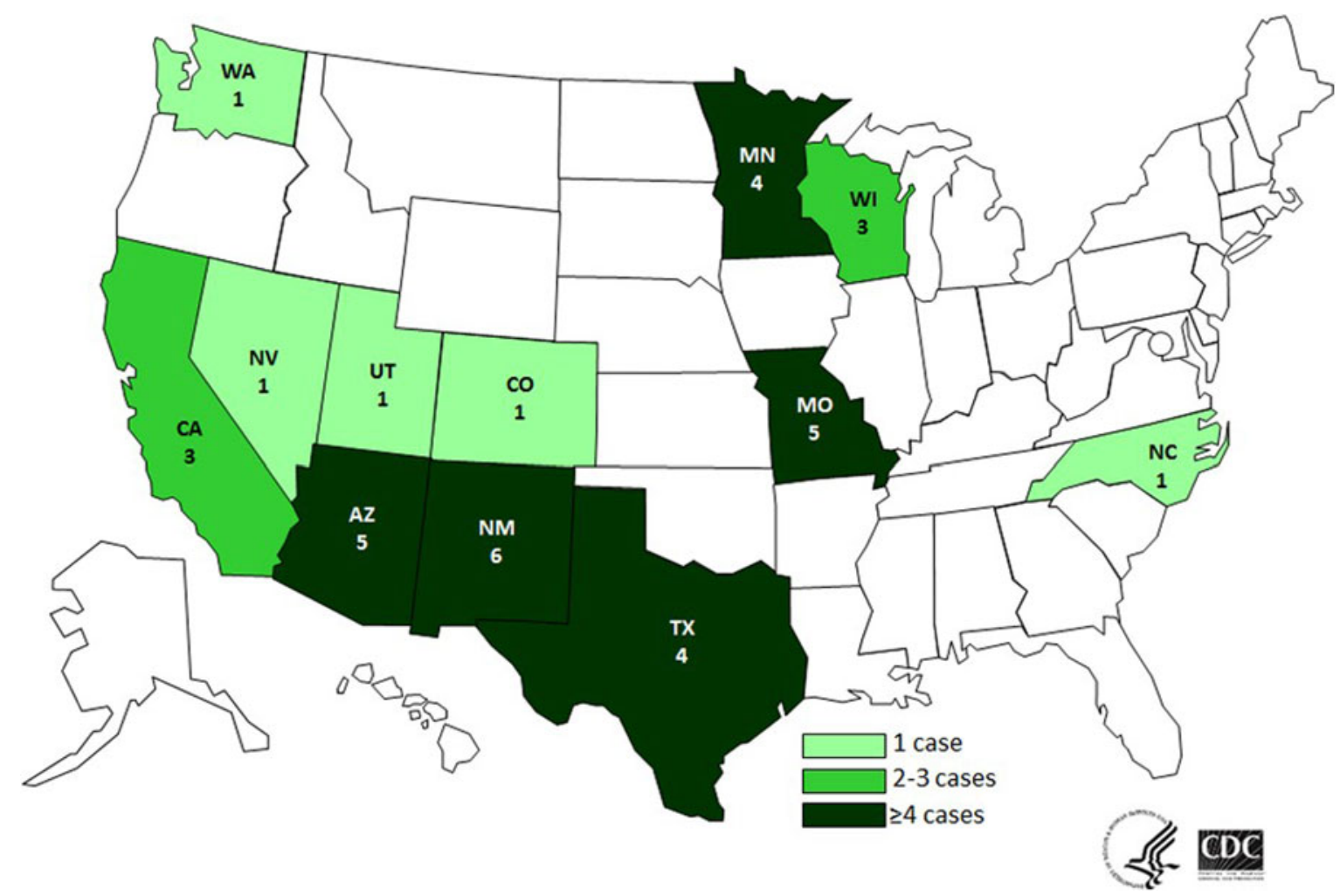

Fig. 1. Number of laboratory-confirmed cases of Listeria monocytogenes infection associated with caramel apples by state of patient residence, United States, 1 October 2014 to 1 February $2015(n=35)$.

and collected 110 environmental samples; seven yielded L. monocytogenes. Six isolates were from food contact surfaces (i.e. polishing brush, drying brushes, conveyor, and inside a wooden bin) and one was from the main packing line floor drain. Although inspectors could not observe packing procedures because seasonal apple packing operations had ended, they observed multiple production line vinyl surfaces with frayed edges, exposed foam padding, and a damaged conveyor belt. FDA, California, and Minnesota officials isolated L. monocytogenes from 30 whole apple samples shipped by Grower A and collected at various points in the distribution chain, as well as one environmental sample from a caramel apple manufacturer.

\section{Subtyping}

Two PFGE patterns were identified in 34 patient isolates. Each PFGE pattern formed a phylogenetically distinct clade by wgMLST. Isolates within each clade were highly related to one another $(<11$ allele differences); a thirty-fifth patient isolate had a third PFGE pattern and grouped closely with one clade (Fig. 3). The two clades aligned with the two illness clusters initially detected by the states and PulseNet, and the clades differed by $>1600$ alleles. Isolates in one clade were multilocus sequence type (ST) 1 and the others were ST382; all isolates were genetic lineage 1 and serotype $4 \mathrm{~b}$. The sequenced $L$. monocytogenes isolates from apples, isolates from Grower A, and the environmental isolate from a caramel apple manufacturer were highly related to patient isolates within both clades by wgMLST.

\section{Control measures}

On 19 December, CDC warned consumers not to eat any commercially produced, prepackaged caramel apples until more specific information could be provided. CDC made this broad recommendation while the traceback investigation was underway and before the contamination scope was known because of listeriosis severity, the long caramel apple shelf-life, and the strong epidemiological link to caramel apple consumption. On 22 December, Grower A recalled whole apples used in caramel apple production after being notified of the epidemiological and traceback evidence. On the 24, 27, and 29 December, three caramel apple manufacturers issued product recalls. On 6 January 2015, 
Table 1. Demographic and clinical characteristics of 35 ill patients with outbreak-associated Listeria monocytogenes infections associated with caramel apples

\begin{tabular}{ll}
\hline \hline Characteristic & Value \\
\hline Median age, years (range) & \\
All cases & $43(0-92)$ \\
Cases not associated with pregnancy & $62(7-92)$ \\
Female sex, $n$ (\%) & \\
All cases & $15(43)$ \\
Cases not associated with pregnancy & $8(33)$ \\
Pregnancy-associated cases, $n(\%)$ & \\
Total & $11(31)$ \\
Pregnant women & $5 / 11(45)$ \\
Neonates & $6 / 11(55)$ \\
Underlying conditions*, $n(\%)$ & $2 / 13(15)$ \\
Cirrhosis or hepatitis & $3 / 13(23)$ \\
Diabetes & $2 / 13(15)$ \\
Heart disease (congenital and acquired) & $4 / 13(31)$ \\
Haemodialysis & $1 / 13(8)$ \\
Myelodysplastic syndrome & $4 / 13(31)$ \\
No underlying condition $\dagger$ & $34(97)$ \\
Hospitalizations, $n(\%)$ & $7(20)$ \\
Reported deaths, $n(\%)$ & $3(9)$ \\
Listeriosis as contributing factor & $1(3)$ \\
Fetal loss & \\
L. monocytogenes source site, $n(\%) \$$ & $26(72)$ \\
Blood & $5(14)$ \\
Cerebrospinal fluid & $1(3)$ \\
Ascitic fluid & $1(3)$ \\
Placenta & $3(8) \S$ \\
Other & \\
\hline \hline &
\end{tabular}

* Excludes pregnancy-associated cases. Complete information on underlying conditions was available for 13 patients. $\uparrow$ Includes three otherwise healthy children between the ages of 5 and 15 years and a 78-year-old female.

\$ One child had a blood isolate matching one outbreak pulsed-field gel electrophoresis (PFGE) pattern and a cerebrospinal fluid isolate matching another outbreak PFGE pattern.

$\S$ Includes specimens from a left arm, sputum, and one site reported as other.

Grower A expanded the recall to include all Gala and Granny Smith apples produced in the 2014 season following environmental sampling results, and CDC narrowed its warning to Grower A's apples.

\section{DISCUSSION}

Commercially produced, prepackaged caramel apples are a newly described listeriosis vehicle. We describe a complex, nationwide foodborne listeriosis investigation in which epidemiological, microbiological, and traceback evidence conclusively demonstrated that commercially produced, prepackaged caramel apples were the source and that the whole apples came from a single grower. This was the largest US listeriosis outbreak since one linked to cantaloupe in 2011 [13]. Caramel apple consumption was significantly higher in outbreak-associated patients than in patients with contemporaneous non-outbreak-associated listeriosis. Traceback investigations converged on a single apple grower in California, and samples from the apple packing facility, one caramel apple production facility, and apples in the distribution chain yielded L. monocytogenes isolates highly related by wgMLST to patient isolates in both outbreak clades. The relatively large size and scope of this outbreak suggests that a critical failure at the facility or an L. monocytogenes introduction occurred, allowing for contamination of whole apples used to make caramel apples. The grower's packing process was the likely contamination source given that $L$. monocytogenes was detected on the packing line and exposed porous surfaces were present.

Three otherwise healthy children developed meningitis. By comparison, only $0 \cdot 6 \%$ of the $>5000$ human L. monocytogenes isolates from the Listeria Initiative were from patients in this age range. Compared with the cantaloupe-associated outbreak [13, 24], this outbreak had a lower median age (43 vs. 78 years) and higher percentage of pregnancy-associated cases $(37 \%$ vs. $4 \%$ ). Age differences in consumption patterns and food preferences or differences in serotype might explain these findings. Although not tested, patients might have been exposed to a relatively high $L$. monocytogenes dose or these L. monocytogenes strains might have been more virulent than others.

Use of wgMLST assisted this investigation by showing that patient isolates within each PFGEdefined cluster were highly related and thus more likely to have a common source. Of note, isolates were not highly related between the two clusters. Subsequently, wgMLST also demonstrated that apple and environmental isolates were highly related to patient isolates. Since September 2013, wgMLST has transformed US listeriosis investigations, helping detect more illness clusters and solve more outbreaks [25]. This investigation also highlighted the importance of 'shoe-leather' epidemiology. After standard forms (LI and NHGQ) failed to identify a likely source, time-intensive open-ended interviews and an iterative interviewing approach were used; an astute epidemiologist who recalled a previous patient's exposure asked specifically about caramel apples in a 


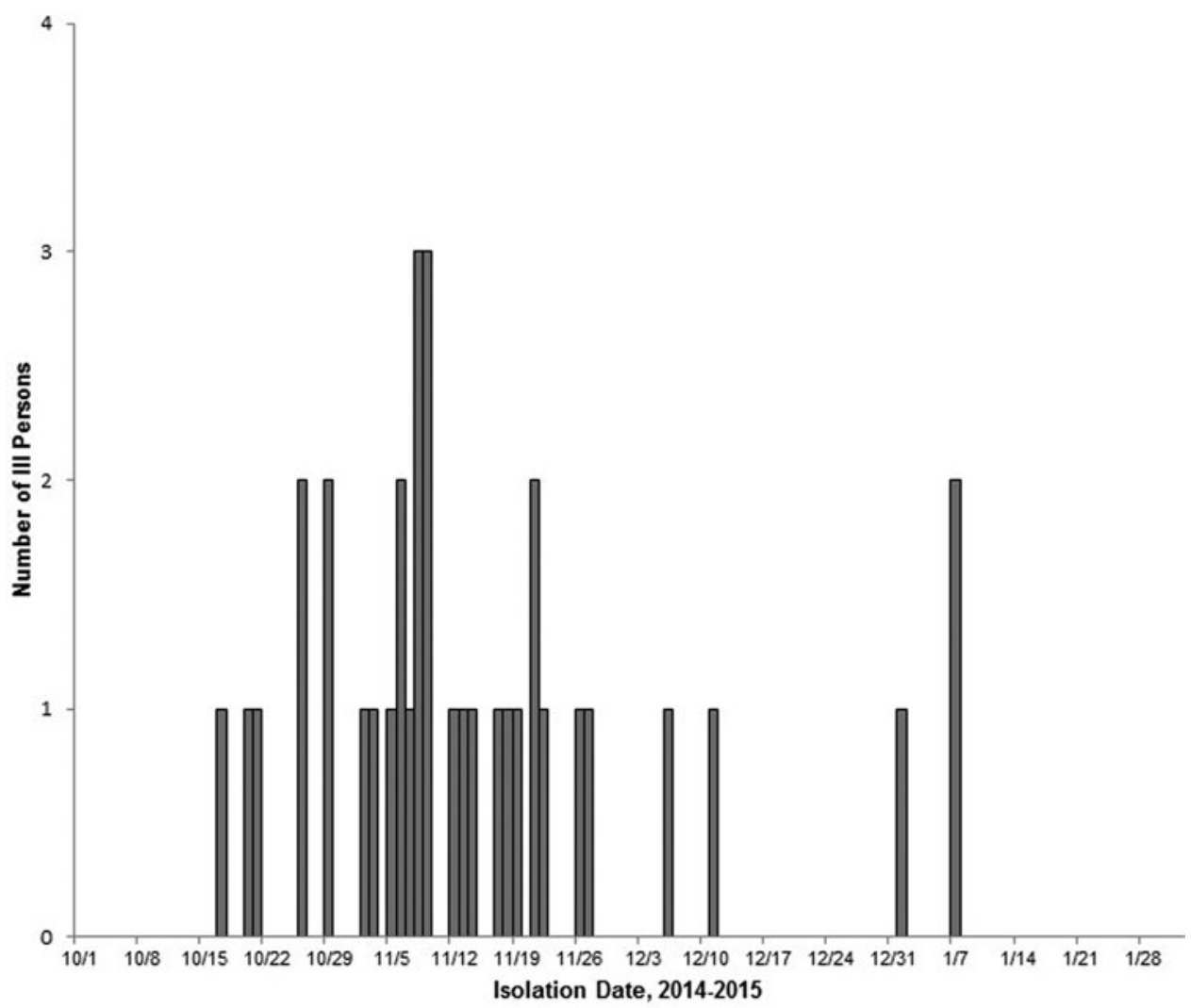

Fig. 2. Dates of Listeria monocytogenes isolation for 35 patients involved in outbreak associated with caramel apples, United States, 1 October 2014 to 1 February 2015.

subsequent interview. Involving the same investigator (s) in several interviews is the key feature that makes this interviewing technique succeed when other methods fail to identify a source [26].

This incident represents the sixth reported US listeriosis outbreak linked to produce since 2008 [9-13]. Whether the emergence of produce as a listeriosis vehicle represents a true increase in produceassociated illnesses or improvements in outbreak detection and investigation is unclear. Although produce is now a well-established listeriosis source, and the first foodborne listeriosis outbreak identified was linked to produce [27], caramel apples were an unexpected food. Neither apples, given their acidity, nor caramel, given its low water activity, were considered likely to support $L$. monocytogenes growth. However, a subsequent inoculation study showed substantial $L$. monocytogenes growth $\left(>3 \log _{10}\right.$ increase within 3 days) on caramel apples with sticks at $25^{\circ} \mathrm{C}$ [28]. Less growth was observed for caramel apples with sticks at $7{ }^{\circ} \mathrm{C}$, and no growth at that temperature was seen in caramel-covered apples without inserted sticks. The authors hypothesized that stick insertion into an apple allows juice to enter the space between the skin and caramel, providing a favourable environment for L. monocytogenes proliferation, particularly at room temperature. We did not collect information on whether patients purchased caramel apples from store shelves or refrigerators or how they stored them at home. At least one grocery store chain involved in the outbreak subsequently stopped selling unrefrigerated caramel apples [29]. Given that other acidic fruit (e.g. peaches, nectarines) were implicated in a small listeriosis outbreak in the summer of 2014, whole apples could be considered a possible $L$. monocytogenes vehicle [11]. Two research findings support this conclusion. First, commercial brush washing of apples immersed in an Escherichia coli solution with sanitizing agents did not reduce $E$. coli populations, likely because the relatively inaccessible stem and calyx areas are harbourage sites [30]. Second, if a pathogen is present, apple wounds or bruises can allow fungal growth that neutralizes apple flesh $\mathrm{pH}$ and facilitates bacterial growth [31].

Consumption of whole apples alone was not significantly associated with illness. Although apples from 


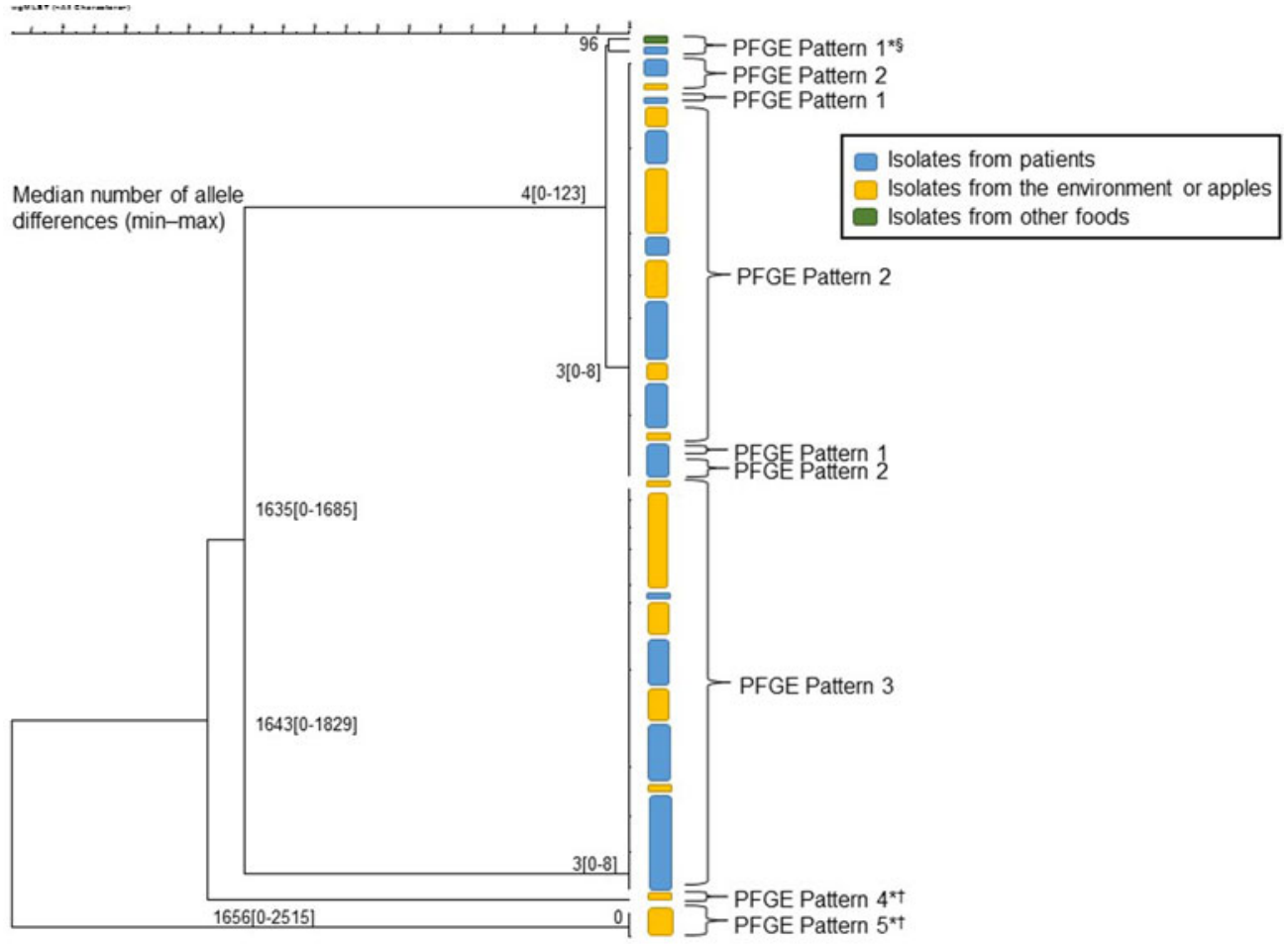

Fig. 3. Dendrogram of whole-genome multilocus sequence typing (wgMLST) results of human, environmental, and produce isolates that match the outbreak strains $(n=50$ isolates). * Isolates not directly related to the outbreak. § Isolates that are indistinguishable from the outbreak PFGE patterns but excluded from the outbreak via wgMLST. $\dagger$ Isolates from an unrelated caramel apple production facility that are not highly related to outbreak-associated isolates. Not all apple isolates underwent wgMLST.

the grower's packing facility bound for caramel apple production and retail yielded L. monocytogenes, there were no differences in apple processing or collection between those intended for caramel apples and those intended for retail. Three outbreak-associated patients who denied eating caramel apples reported eating whole or pre-sliced apples. However, $\sim 60 \%$ of the US population report eating apples during a given week [23] and it was not possible to determine the origin of the apples eaten by these patients, so it is not known whether they came from the California grower or if they were the source of the patients' illnesses.

This outbreak highlights gaps in the understanding of microbiological risks associated with tree fruit production, the importance of preventive measures to minimize contamination of ready-to-eat produce items with L. monocytogenes. FDA's Produce Safety Rule under the U.S. Food Safety Modernization Act could help reduce the listeriosis risk from fruit by requiring that non-exempt producers inspect, maintain, clean, and sanitize all food contact surfaces and take other preventive actions [32]. Caramel apple manufacturers should consider package labels recommending product refrigeration, and retailers and consumers should consider refrigerating prepackaged caramel apples and follow use-by dates to maintain quality. For future public health investigations, listeriosis questionnaires should include more produce and seasonal or holiday items questions, including caramel apples, and investigators should perform single-interviewer open-ended interviews when a food vehicle is not readily identified.

\section{ACKNOWLEDGEMENTS}

We thank those involved in the Listeria Outbreak Investigation Team for their assistance with data collection, interviewing, scientific advice, and reviewing the manuscript:

Kevin McClaran, BS (Texas Department of State Health Services, Lubbock, Texas); Venessa Cantu, MPH (Texas Department of State Health Services, Austin, Texas); Greg Leos, MPH, CPH (Texas Department of State Health Services, Austin, Texas); Irina Cody, MPH (Texas Department of State Health Services, Austin, Texas); Akiko Kimura, MD 
(California Department of Public Health, Los Angeles, California); Hilary Rosen, MPH (California Department of Public Health, Los Angeles, California); Michael Needham, MPH (California Department of Public Health, Sacramento, California); Virginia Philips, BA (Missouri Department of Health and Senior Services, St. Louis, Missouri); Jo Ann Rudroff (Missouri Department of Health and Senior Services, Jefferson City, Missouri); Kirk Smith, DVM, MS, PhD (Minnesota Department of Health, St. Paul, Minnesota); Rachel Klos, DVM, MPH (Wisconsin Department of Health Services, Madison, Wisconsin); Justin Kohl, MPH (Wisconsin Department of Health Services, Madison, Wisconsin); Nicole Lee, MPH (North Carolina Department of Health and Human Services, Raleigh, North Carolina); Vanessa M. Greene, RN (North Carolina Division of Public Health, Raleigh, North Carolina); Rick Sowadsky, MSPH (Nevada Division of Public and Behavioral Health, Carson City, Nevada); Nisha Alden, MPH (Colorado Department of Public Health and Environment, Denver, Colorado); Beth Melius, RN, MN, MPH (Washington State Department of Health, Shoreline, Washington); Capt. Brian Johnson, REHS, MPH (Navajo Area Indian Health Service, Saint Michaels, Arizona); Diane MacDonald, MHSc (Public Health Agency of Canada, Ottawa, Ontario, Canada); Errol Strain, $\mathrm{PhD}$ (Food and Drug Administration, College Park, Maryland); David Melka (Food and Drug Administration, College Park, Maryland); Monique Salter, MPH (Food and Drug Administration, College Park, Maryland); Joseph Blankenship, MPH (Food and Drug Administration, College Park, Maryland); Rachel Goeriz Pearson, PhD (Food and Drug Administration, College Park, Maryland); Ian T. Williams, PhD, MS (Centers for Disease Control and Prevention, Atlanta, Georgia); Karen Neil, MD, MSPH (Centers for Disease Control and Prevention, Atlanta, Georgia); Katie Roache, BS (Centers for Disease Control and Prevention, Atlanta, Georgia); Laura Burnworth, MPH (Centers for Disease Control and Prevention, Atlanta, Georgia); Mandip Kaur, MPH (Centers for Disease Control and Prevention, Atlanta, Georgia);

The opinions expressed by the authors contributing to this journal do not necessarily reflect the opinions of the Centers for Disease Control and Prevention, the Food and Drug Administration, or the institutions with which the authors are affiliated.

The authors have no funding to report.

\section{DECLARATION OF INTEREST}

None.

\section{REFERENCES}

1. Scallan E, et al. Foodborne illness acquired in the United States-major pathogens. Emerging Infectious Diseases 2011; 17: 7-15.

2. Centers for Disease Control and Prevention. Vital signs: Listeria illnesses, deaths, and outbreaks - United States, 2009-2011. Morbidity and Mortality Weekly Report 2013; 62: 448-452.

3. Painter J, Slutsker L. Listeriosis in humans. In: Ryser ET, Marth EH, eds. Listeria, Listeriosis and Food Safety, 3rd edn. Boca Raton, Florida: Taylor and Francis Group, 2007. pp. 85-110.

4. Swaminathan B, Gerner-Smidt P. The epidemiology of human listeriosis. Microbes Infection 2007; 9: 1236 1243.

5. Gottlieb SL, et al. Multistate outbreak of listeriosis linked to turkey deli meat and subsequent changes in US regulatory policy. Clinical Infectious Diseases 2006; 42: 29-36.

6. Jackson KA, et al. Multistate outbreak of Listeria monocytogenes associated with Mexican-style cheese made from pasteurized milk among pregnant, hispanic women. Journal of Food Protection 2011; 74: 949-953.

7. Mead PS, et al. Nationwide outbreak of listeriosis due to contaminated meat. Epidemiology and Infection 2006; 134: 744-751.

8. de Valk $\mathbf{H}$, et al. Two consecutive nationwide outbreaks of listeriosis in France, October 1999-February 2000. American Journal of Epidemiology 2001; 154: 944-950.

9. Garner D, Kathariou S. Fresh produce-associated listeriosis outbreaks, sources of concern, teachable moments, and insights. Journal of Food Protection 2016; 79: 337-344.

10. Centers for Disease Control and Prevention. Wholesome Soy Products, Inc. Atlanta: Sprouts and investigation of human listeriosis cases (Final update), 27 January 2015 (http://www.cdc.gov/listeria/outbreaks/bean-sprouts-1114/index.html). Accessed 1 November 2015.

11. Jackson BA, et al. Listeriosis associated with stone fruit-United States, 2014. Morbidity and Mortality Weekly Report 2015; 64: 282-283.

12. Gaul LK, et al. Hospital-acquired listeriosis outbreak caused by contaminated diced celery - Texas, 2010. Clinical Infectious Diseases 2013; 56: 20-26.

13. McCollum JT, et al. Multistate outbreak of listeriosis associated with cantaloupe. New England Journal of Medicine 2013; 10: 944-953.

14. Food and Drug Administration. Crunch pak issues voluntary recall of limited quantities of crunch pak brand apple slices because of possible health risk, 2013. (http://www.fda.gov/Safety/Recalls/ucm375135.htm). Accessed 16 February 2016.

15. Centers for Disease Control and Prevention. Outbreaks of Escherichia coli O157:H7 infection and cryptosporidiosis associated with drinking unpasteurized apple 
cider x-Connecticut and New York, October 1996. Morbidity and Mortality Weekly Report 1997; 46: 4-8.

16. Gerner-Smidt P, et al. PulseNet USA: A five-year update. Foodborne Pathogens and Disease 2006; 3: 9-19.

17. Swamanathan B, et al. PulseNet: the molecular subtyping network for foodborne bacterial disease surveillance, United States. Emerging Infectious Diseases 2001; 7: 382-389.

18. Halpin JL, et al. Re-evaluation, optimization, and multilaboratory validation of the PulseNet-standardized pulsedfield gel electrophoresis protocol for Listeria monocytogenes. Foodborne Pathogens and Disease 2010; 7: 293-298.

19. Sabat AJ, et al. Overview of molecular typing methods for outbreak detection and epidemiological surveillance. Eurosurveillance 2013; 18.

20. PulseNet International. PulseNet International the International Molecular Subtyping Network for Foodborne Disease Surveillance, 2015 (http://www. pulsenetinternational.org/). Accessed 29 September 2015.

21. Centers for Disease Control and Prevention. National enteric disease surveillance: the Listeria initiative. 2011 (http://www.cdc.gov/listeria/pdf/ListeriaInitiativeOverview_ 508.pdf). Accessed 12 October 2015.

22. Centers for Disease Control and Prevention. Foodborne disease outbreak investigation and surveillance tools. 2015 (http://www.cdc.gov/foodsafety/outbreaks/surveillance-reporting/investigation-toolkit.html). Accessed 29 September 2015.

23. Centers for Disease Control and Prevention. Foodborne diseases active surveillance network (FoodNet) population survey atlas of exposures, 2006-2007. 2008 (http:// www.cdc.gov/foodnet/PDFs/FNExpAt103022011.pdf). Accessed 29 September 2015.

24. Centers for Disease Control and Prevention. Multistate outbreak of listeriosis linked to whole cantaloupes from Jensen farms, Colorado. 2012 (http://www.cdc. gov/listeria/outbreaks/cantaloupes-jensen-farms/082712/ index.html). Accessed 30 October 2015.

25. Jackson BR, et al. Implementation of nationwide realtime whole-genome sequencing to enhance listeriosis outbreak detection and investigation. Clinical Infectious Diseases 2016. 63: 380-386.

26. Mody RK, et al. Outbreak of Salmonella enterica serotype I 4,5,23:1:- infections: the challenges of hypothesis generation and microwave cooking. Epidemiology and Infection 2014; 142: 1050-1060.

27. Schlech WF 3rd, et al. Epidemic listeriosis - evidence for transmission by food. New England Journal of Medicine 1983; 308: 203-206.

28. Glass KA, et al. Growth of Listeria monocytogenes within a caramel-coated apple microenvironment. mBio 6: e01232-15.

29. The Wall Street Journal. Kroger pulls caramel apples after study on Listeria threat. New York, 2015 (http:// www.wsj.com/articles/kroger-pulls-caramel-apples-afterstudy-on-listeria-threat-1444947940). Accessed 16 October 2015.

30. Annous BA, et al. Efficacy of washing with a commercial flatbed brush washer, using conventional and experimental washing agents, in reducing populations of Escherichia coli on artificially inoculated apples. Journal of Food Protection 2001; 654: 159-163.

31. Riordan DC, Sapers G. The survival of Escherichia coli O157:H7 in the presence of Penicillium expansum and Glomerella cingulate in wounds on apple surfaces. Journal of Food Protection 2000; 12: 1637-1642.

32. Food and Drug Administration. FSMA final rule on produce safety. Silver Spring, Maryland (http://www.fda. gov/Food/GuidanceRegulation/FSMA/ucm334114.htm). Accessed 30 October 2015. 